\title{
preprints
}

Editorial

\section{One Thousand Preprints Online}

\section{Preprints Editorial Office}

MDPI, St. Alban-Anlage 66, 4052 Basel, Switzerland; E-Mail: info@preprints.org;

Tel.: +41-61-683-7734

Preprints is delighted to have passed the milestone of one thousand preprints online. This is a significant step for the platform, which was launched in May last year with the objective of speeding up the process of communicating research across all disciplines. We are proud to be the first preprint server to cover all disciplines.

\section{Fulfilling Preprints' Aims}

Preprints is a platform to disseminate research at an early stage, before peer review. It was established by MDPI on a not-for-profit basis. The stated aim in our initial editorial [1] was to make preprints discoverable, sharable and citable. While there is still more to do, we have made significant progress. Preprints was the first to use Crossref's new schema designed specifically for preprints, meaning articles online can be cited by standard mechanisms and are assigned a unique digital object identifier (DOI). Our preprints appear on several platforms apart from our own website, including:

- Google Scholar https://scholar.google.com/scholar?as ylo=2016\&hl=en\&as publication=preprints

- Academic Karma http://academickarma.org/preprints.org

- SHARE, from the Open Science Foundation https://share.osf.io/discover?sort=-date created\&sources=Preprints.org

We receive approximately 30,000 page views per month and maintain a Twitter account at $@$ Preprints_org to highlight some of the latest papers. Many individual preprints have received hundreds of page views and downloads.

\section{Integrating the Research Community}

In the next phase, we want to continue to build on progress so far and work more closely with the research community. The advisory board has already been expanded to 96 active researchers across a range of disciplines. Currently, we are working on:

- Involving researchers in the initial review process;

- Integrating ORCID (https://orcid.org);

- Enabling submission in languages other than English.

Preprints is also partnering with Publons (https://publons.com/home/) so that reviewers who post their comments on articles can keep a verified record of every preprint reviewed. This will be an important step to encourage more comments and give proper recognition to those who give valuable feedback to authors. 


\section{Efficiency}

From the outset, we aimed to assess preprints within 24 hours and we are pleased to have achieved this goal in almost all cases. We have also optimized our initial screening process to ensure submitted manuscripts comply with the requirements we have. However, our submission form still looks like a journal submission and we think we can make it even simpler: authors simply upload the file and our software and editors will extract the data. Look out for changes in the coming weeks.

\section{Fulfilling the Potential of Preprints}

Preprints is dedicated to speeding up the dissemination of science and the putting reporting of research into the hands of those producing it. The concept of preprints has the potential to significantly change how research is reported and evaluated in a way that is beneficial to all stakeholders: authors control when their work is made public, readers get to see the latest research as early as possible, reviewers can have their contribution publicly recognized and journal editors can receive papers that have already been improved by feedback. We are pleased to be a part of that change and look forward to further expansion of Preprints.

\section{Bibliography}

1. Preprints Editorial Office. Introducing Preprints: A Multidisciplinary Open Access Preprint Platform. Preprints 2016, 2016050001; doi: 10.20944/preprints201605.0001.v1. 